\title{
Superconducting plasmonics and extraordinary transmission
}

\author{
A. R. Buckingham ${ }^{\text {I }}$ A. Tsiatmas ${ }^{2}$, V. A. Fedotov ${ }^{2}$, Y. Chen ${ }^{3}$, P. A. J. de Groot ${ }^{\text {I }}$ and N. I. Zheludev ${ }^{2}$ \\ ${ }^{1}$ School of Physics and Astronomy, University of Southampton, Southampton, SO17 1BJ, UK \\ ${ }^{2}$ Optoelectronics Research Centre, University of Southampton, Southampton, SO17 1BJ, UK \\ ${ }^{3}$ Rutherford Appleton Laboratory, Harwell Science and Innovation Campus, Didcot, OX11 OQX, UK
}

Superconductors may be considered as plasmonic media, exhibiting a resistance dominated by the kinetic motion of electrons and a negative dielectric constant. In metamaterials Joules losses are significant and substantially reduce achievable Q-factors. The use of superconductors in metamaterials permits the control and reduction of Joule losses, opening the opportunity for achieving low-loss, high Q-factor metamaterials.

We report on free-space millimetre-wave measurements of the temperature dependent transmission in high temperature superconducting (HTS) metamaterial structures. HTS films of $\mathrm{YBa}_{2} \mathrm{Cu}_{3} \mathrm{O}_{7.5}$ have been lithographically structured into arrays of both asymmetric split rings (ASR) and holes. Experimental data show that Fano resonances in the ASR arrays become substantially stronger as the superconducting critical temperature $\left(T_{C}\right)$ is reached. This is in good agreement with data derived from a two-fluid model (Figure 1). We conclude this provides a route towards high Qfactor spectral filters in the millimetric-wave regime [1]. Temperature dependent extraordinary transmission peaks are observed in the hole array. The peaks dramatically increase in amplitude as $T_{C}$ is reached and we observe a Q-factor exceeding 250 which is attributed to plasmonic interactions (Figure 2). Our data show that extraordinary transmission does not require surface plasmons to exist, but is further enhanced in the plasmonic regime of the media [2]. We also report on recent measurements on $\mathrm{Nb}$ based superconducting metamaterials where losses may be reduced further still, and on arrays of interacting superconducting disks where the enhancement of the incident electromagnetic field is able to break superconductivity leading to a non-linear metamaterial.

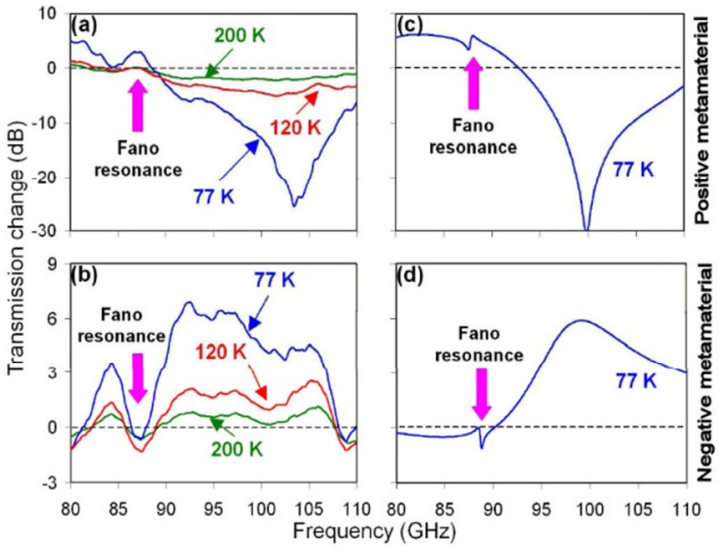

Figure 1: Experimental verification of Fano resonances in positive (graph a) and negative (graph b) ASR metamaterial arrays, and complimentary two-fluid based computational model (graphs c and d).

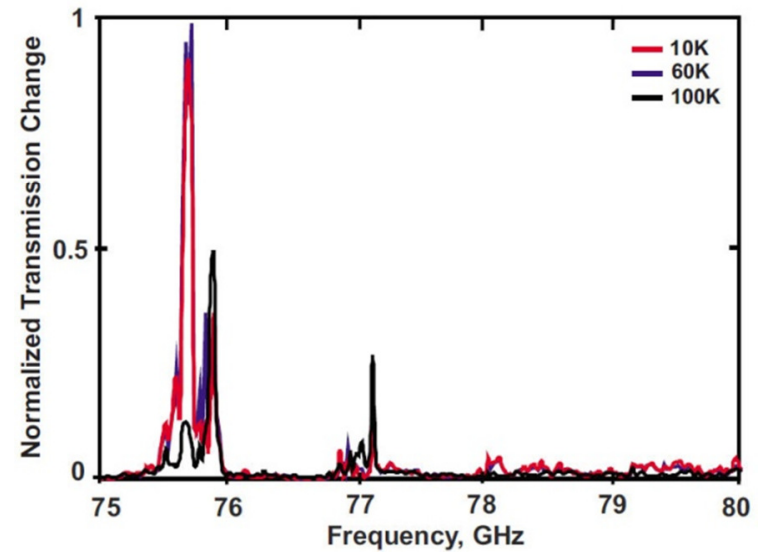

Figure 2: Temperature dependent extraordinary transmission peaks in a hole array fabricated in a HTS film.

[1] V.A. Fedotov, A. Tsiatmas, J. H. Shi, R. Buckingham, P. de Groot, Y. Chen, S. Wang and N.I. Zheludev, Opt. Exp. 18 (9), 9015 (2010)

[2] A. Tsiatmas, A. R. Buckingham, V. A. Fedotov, S. Wang, Y. Chen, P. A. J. de Groot and N. I. Zheludev, Appl. Phys. Lett. 97 (1), 111106 (2010) 